\title{
PROTHROMBOTIC STATUS IN ACTIVE- AND ACUTE STAGES OF CANINE MONOCYTIC EHRLICHIOSIS
}

\author{
Adnan Ayan $^{1 *}$, Kerem Ural ${ }^{2}$ \\ I*Department of Genetics, Faculty of Veterinary Medicine, Van Yuzuncu Yil University, Tusba, Van, Turkey; \\ ${ }^{2}$ Department of Internal Medicine, Faculty of Veterinary Medicine, Adnan Menderes University, \\ Isikli, Aydın, Turkey; \\ *Corresponding Author, Adnan AYAN, e-mail: adnanayan@yyu.edu.tr;
}

Received June 2019; Accepted July 2019; Published September 2019;

DOI: https://doi.org/10.31407/ijees9320

\begin{abstract}
Biomarkers in an attempt to determine prothrombotic condition alterations, for the vast majority by use of D-dimer, has long been elucidated retrospectively. D-dimer, a well known breakdown/degradation product of cross-linked fibrin, has been the subject of several researches. On the other hand to the present authors knowledge, to those of different stages of Canine Monocytic Erlichiosis (CME), D-dimer levels have not been analyzed, which should thoroughly effect therapeutic scenario. The aim of this study was to measure D-dimer concentrations and assess their value in the diagnosis of CME. Therefore D-dimer analyses by use of Wondfo Finecare Fluorescent Immunoassay were performed in four groups of dogs; (i) 8 dogs with acute CME, ii) 9 dogs with active CME infection, iii) exposured dogs $(n=8)$ then were compared to those of healthy dogs ( $n=9$ dogs as iv) control group. The D-dimer range in clinically healthy dogs was $<0.1 \mathrm{mg} / \mathrm{L}$. In the present study D-dimer levels were detected as follows: $0,06 \pm 0,10,3,20 \pm 3,05,4,04 \pm 3,94$ and $0,06 \pm 0,07 \mathrm{mg} / \mathrm{dl}$ for control, acute infected, active infected and exposured dogs with a statistical significance $(\mathrm{p}<0.01)$ as shown in table In both infected groups, D-dimer levels increased with clinical evidence of disease. D-dimer concentration may be considered as an indicator for disease activity during acute/active disease condition and may be useful as a potential biochemical marker.
\end{abstract}

Keywords: Acute stages, Canine Monocytic Ehrlichiosis, Prothrombotic status. 